03

\title{
Расчет сопротивления и теплоотдачи шара в ламинарном и сильнотурбулентном потоках газа
}

\author{
(C) Н.Н. Симаков
}

Ярославский государственный технический университет, 150023 Ярославль, Россия

e-mail: nik_simakov@mail.ru

(Поступило в Редакцию 13 мая 2015 г.

В окончательной редакции 30 марта 2016 г)

При сильной турбулентности набегающего на шар газового потока может возникать ранний кризис сопротивления. Для исследования его влияния на теплоотдачу шара газу выполнен численный эксперимент, в котором моделировалось обтекание шара свободным газовым потоком с меньшей, чем у шара, температурой в двух случаях. В первом случае поток был ламинарным, во втором - сильнотурбулентным. Турбулентность учитывалась значением кинематического коэффициента турбулентной вязкости, гораздо (до 2000 раз) большим, чем у физической вязкости. Результаты расчетов показали, что возникновение раннего кризиса сопротивления при числах Рейнольдса Re порядка 100, выражающееся в значительном (в 4-7 раз) уменьшении гидродинамической силы и коэффициента сопротивления $C_{d}$ шара, сопровождается также кризисом теплоотдачи шара газу с уменьшением числа Нуссельта $\mathrm{Nu}$ в 3-6 раз.

\section{Введение. Ранний кризис сопротивления шара}

Во многих технологических процессах для повышения интенсивности тепломассообмена фаз за счет увеличения межфазной поверхности используется распыливание жидкости в газе, например, с помощью форсунок.

При этом образуются капли со средним диаметром примерно $d \sim 10^{-4} \mathrm{~m}$. При таких размерах и большом различии динамических коэффициентов вязкости жидкости капель и обтекающего их газа (для воды и воздуха примерно в 60 раз) деформацией капель и внутренним течением жидкости в них можно пренебречь, рассматривая их как твердые шарики [1].

При расчете процессов с распыливанием жидкости, чтобы вычислить гидродинамическую силу сопротивления капли

$$
F=C_{d} S \rho V^{2} / 2
$$

при ее относительном движении в газе со скоростью $V=V_{\infty}\left(V_{\infty}-\right.$ скорость газа вдали от капли $)$, необходимо знать значения коэффициента $C_{d}$ гидродинамического сопротивления.

Здесь и ниже обозначено $S=\pi d^{2} / 4$ - площадь миделева сечения сферической капли, $d-$ ее диаметр, $\rho$ - плотность газа, $\mu-$ динамический и $v=\mu / \rho-$ кинематический коэффициенты вязкости газа.

Для обтекания шара ламинарным потоком при числах Рейнольдса $\operatorname{Re}=V d \rho / \mu \ll 1$ известна формула Стокса

$$
C_{d}=24 / \operatorname{Re} .
$$

А для случаев обтекания шара в переходном диапазоне $2<\operatorname{Re}<700$ известна зависимость Клячко

$$
C_{d}=24 / \operatorname{Re}+4 / \operatorname{Re}^{1 / 3}
$$

которая в указанном диапазоне достаточно хорошо аппроксимирует экспериментальные данные, обобщенные кривой Рэлея [2,3].

В работах [4,5] по данным эксперимента установлено (рис. 1), что в сильнотурбулентном двуфазном потоке диаметром $D$ и средней скоростью $\langle V\rangle$, для которого $\operatorname{Re}_{1}=\langle V\rangle D \rho / \mu \sim 10^{5}$, величина $C_{d}$ для капель, обтекаемых при $\operatorname{Re} \sim 100$, может уменьшаться в 4-7 раз по сравнению с общеизвестными значениями, определяемыми формулой (3) для ламинарного набегающего

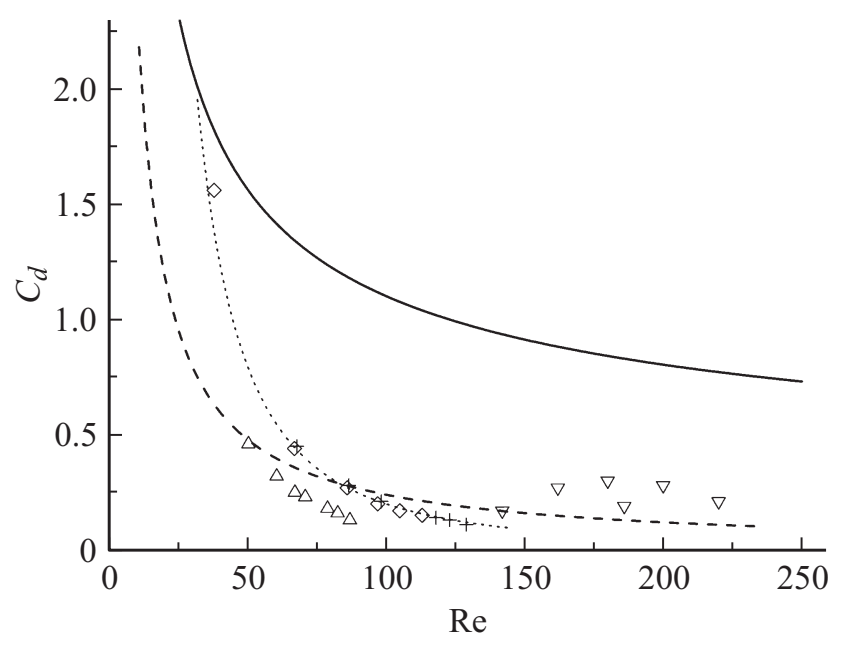

Рис. 1. Зависимости коэффициента сопротивления $C_{d}$ шара от числа Рейнольдса Re: символы - данные эксперимента с каплями воды в факеле форсунки при давлениях, $P: \diamond-5$, $+-3, \Delta-9$ bar, пунктирная кривая - аппроксимация части этих данных формулой $C_{d}=2000 / \mathrm{Re}^{2}$; сплошная кривая - по формуле (3), штриховая линия - по формуле (2), $\nabla$ - данные эксперимента [3] с шариком, обдуваемым струей в конфузоре. 
потока. Такой же ранний кризис сопротивления на одиночном твердом шарике не наблюдался при его обтекании свободной газовой струей, но возникал в струе, протекавшей через конфузор [6].

Заметим, что отображающие результаты эксперимента символы на рис. 1 расположены близко к штриховой кривой, соответствующей формуле Стокса (2).

Предположение о возможном значительном влиянии геометрии набегающего потока на гидродинамическое сопротивление обтекаемого тела в численном эксперименте не подтвердилось [7].

Другим объяснением причины раннего кризиса сопротивления сферической частицы была гипотеза о влиянии сильной турбулентности газового потока, которую конфузор по сравнению со свободной струей мог еще больше повысить и сделать достаточной для возникновения раннего кризиса $[6,7]$.

Это предположение подтвердилось численным экспериментом, в котором моделировалось обтекание шара свободным газовым потоком как ламинарным, так и сильнотурбулентным [8].

Как известно, между явлениями переноса импульса, тепла и массы в неоднородном потоке существует аналогия, согласно которой профили скоростей, концентраций примеси и температур в разных сечениях потока могут обладать некоторым подобием $[9,10]$. С учетом этой аналогии кризис сопротивления шара в сильнотурбулентном потоке с неизбежностью должен влиять на тепло- и массообмен шара с газом. Представлялось интересным оценить это влияние каким-либо образом, например, путем численного моделирования обтекания шара газом с учетом теплообмена.

Теоретическая оценка этого влияния была сделана в работе [11], где было показано, что ранний кризис сопротивления шара должен сопровождаться уменьшением коэффициента теплоотдачи шара газу в 3.5-5.8 раза.

Цель настоящей работы - исследовать методом численного моделирования и подтвердить влияние сильной турбулентности набегающего на шар газового потока (с возникновением раннего кризиса сопротивления) на теплоотдачу нагретого шара газу с меньшей температурой.

\section{1. Моделирование обтекания шара ламинарным потоком газа с учетом их теплообмена}

Математическая модель течения газа с малыми (по сравнению со скоростью звука $V_{s}$ ) скоростями $V$ включала в себя уравнение неразрывности

$$
\partial \rho / \partial t+\operatorname{div}(\rho \mathbf{V})=0
$$

и уравнение Навье-Стокса

$$
\partial \mathbf{V} / \partial t+(\mathbf{V} \nabla) \mathbf{V}=-\nabla P / \rho+v \Delta \mathbf{V}
$$

Для учета теплообмена между шаром и газом, имеющим температуру $T$, меньшую, чем шар, математическая модель дополнялась „общим уравнением переноса тепла“ [9], также называемым „уравнением баланса тепла“ $[10]$, которое в данном случае было приведено к виду

$$
\rho c_{v} d T / d t=-P \operatorname{div} \mathbf{V}+\operatorname{div}(\lambda \nabla T)+\Phi_{d} .
$$

Для связи давления и плотности газа по аналогии с работами $[7,8]$ использовалось соотношение

$$
d P=\gamma P / \rho d \rho
$$

которое следует из уравнения адиабаты Пуассона $P / \rho^{\gamma}=$ const, где $\gamma-$ постоянная адиабаты. В наших расчетах использовалось значение $\gamma=1.40$, как у воздуха.

В книге [10] предлагалось вместо уравнения Пуассона использовать уравнение Клапейрона-Менделеева

$$
P=\rho R T / M,
$$

связывающее параметры состояния $P, \rho$ и $T$ идеального газа.

Однако стоит заметить, что уравнение (8) справедливо для газа, находящегося в состоянии термодинамического равновесия. Чего нельзя сказать про движущийся газ с переменными по пространству скоростями и температурами, для которого равновесие можно считать только локальным [12]. Поэтому формулу (8) можно применять для связи средних по времени значений величин $P, \rho$ и $T$ в данной точке пространства, но не для определения связи их дифференциалов.

В уравнениях (4)-(8) и далее использованы также обозначения $R$ - универсальная газовая постоянная, $c_{v}$ - удельная теплоемкость газа при постоянном объеме, $M$ - молярная масса, $\lambda$ и $a=\lambda /\left(\rho c_{v}\right)-$ коэффициенты теплопроводности и температуропроводности, $v=\mu / \rho-$ кинематический коэффициент вязкости газа. В расчете данные о физических свойствах газа принимались, как для воздуха.

В формуле (6) последний член $\Phi_{d}$ учитывает диссипацию механической энергии газа в тепло. Согласно источникам [9] и [10], при малых скоростях течения и степени сжатия газа (когда можно положить $\operatorname{div} \mathbf{V} \approx 0$ ) этот член можно представить в виде

$$
\Phi_{d}=\mu / 2\left(\partial V_{i} / \partial x_{k}+\partial V_{k} / \partial x_{i}\right)^{2} .
$$

При небольших различиях температур шара и обтекающего его газа изменением коэффициентов $\mu$ и $\lambda$ можно пренебречь. Как показали расчеты, влияние члена $\Phi_{d}$ на распределения скоростей и температур газа при этом также мало. Тогда уравнение (6) приводится к виду

$$
\partial T / \partial t=-\mathbf{V} \nabla T-0.4 T \operatorname{div} \mathbf{V}+a \Delta T .
$$

С учетом формулы (8) и данных для воздуха здесь использованы соотношения $P /\left(\rho c_{v}\right)=R T /\left(M c_{v}\right) \approx 0.4 T$. 
Уравнения (4), (5) и (10) сначала записывались для сферической системы координат, а затем уже представлялись в конечно-разностной форме. В двумерной декартовой системе координат расчетная область имела форму полукольца. А в сферической (полярной) системе координат - форму прямоугольника с размерами 44 точки по радиусу $r_{j}=j h$ (номера слоев $j=10-53$ ) и 26 точек $(i=0-26)$ по угловой координате - полярному углу $\theta_{i}=0-\pi(\mathrm{rad})$ между полярной осью $\mathrm{OZ}$ и радиус-вектором $\mathbf{r}$ данной точки. Центр симметрии области совпадал с центром шара радиусом $\mathrm{R}=10 h$, где $h$ - шаг сетки по $r$. Направление полярной оси совпадало с направлением набегающего на шар газового потока, однородного вдали от шара.

В выбранной системе координат уравнения (4), (5) и (10) переписывались в конечно-разностной форме с использованием явной схемы Лакса-Вендроффа [13]. Аппроксимация конвективных членов и устойчивость этой схемы были исследованы с помощью спектрального признака в той же работе [13]. Такая же схема использовалась автором настоящей статьи ранее в работах $[7,8]$. Она является устойчивой при ограничении шага $\tau$ по времени условием Куранта-Фридрихса-Леви в виде

$$
\tau<h /\left(\left(V+V_{s}\right) 2^{1 / 2}\right)
$$

где $V=\left(V_{\theta}^{2}+V_{r}^{2}\right)^{1 / 2}$ - модуль скорости газа, $V_{\theta}$ и $V_{r}$ - проекции скорости газа на оси координат, $V_{s}=$ $=(\gamma P / \rho)^{1 / 2}-$ скорость звука в газе.

В сочетании со схемой Лакса-Вендроффа аппроксимация вязких (диффузионных) членов уравнений (5) и (10) модели проводилась по явной схеме первого порядка точности [13], для которой в случае двумерной сетки условие устойчивости имеет вид

$$
\tau \leq h^{2} /(4 v),
$$

где вместо $v$ может быть коэффициент $a$ температуропроводности газа из формулы (10).

Для обеспечения устойчивости разностной схемы в целом необходимо одновременное выполнение обоих условий (11) и (12) [13], из которых второе в данном случае оказалось более сильным.

Разностные аналоги дифференциальных уравнений (4), (5) и (10) дополнялись соответствующими граничными условиями и решались численно до установления стационарного решения. В частности, на поверхности шара граничным условием было $\mathbf{V}=0$ - обращение скорости газа в нуль. На входной границе области $(j=53, i=13-26)$ плотность $\rho$ и температура газа поддерживались постоянными $\rho=$ const, $T=\mathrm{const}=T_{1}$. На поверхности шара температура тоже поддерживалась постоянной, но другой $T=\mathrm{const}=T_{2}=T_{1}+\Delta T \quad \mathrm{c}$ небольшим различием $\Delta T \ll T_{1}$.

По вычисленному полю температур газа вблизи поверхности шара вычисляли поток тепла с поверхности шара площадью $f$ к обтекающему его газу

$$
q=d Q / d t=-\lambda \int \partial T / \partial z d f .
$$

Затем вычисляли коэффициент $\alpha$ теплоотдачи шара газу и значение числа Нуссельта $\mathrm{Nu}$ по формулам

$$
\alpha=q /(f \Delta T), \quad \mathrm{Nu}=\alpha d / \lambda .
$$

По вычисленному полю скоростей и давлений газа интегрированием напряжений по поверхности шара находили действующую на него силу

$$
F=\int\left(-P \cos \theta+3 / 2 v \rho V_{\infty} \sin ^{2} \theta / R\right) d f .
$$

Затем вычисляли коэффициент сопротивления $C_{d}$ шара, выразив его из формулы (1).

\section{2. Результаты численного эксперимента}

По вышеописанному алгоритму на языке Паскаль была составлена программа, с использованием которой выполнялись расчеты.

Рассчитанные профили температуры газа представлены на рис. 2-5. На рис. 2 и 3 соответственно представлены радиальные профили температуры газа и профили ее изменения по полярному углу $\theta_{i}$ для стоксовского режима обтекания шара ламинарным потоком газа при $\operatorname{Re}=0.125$. На рис. 4 и 5 - то же для переходного режима при $\mathrm{Re}=128$.

На рис. 2 очевидно, что в стоксовском режиме радиальные профили температуры $\left(T\left[\theta_{i}, r_{j}\right]-T_{1}\right) / \Delta T=$ $=t[i, j]$ газа (при $i=\mathrm{const}$ ) немного отличаются для разных полярных углов $\theta_{i}$ и напоминают о центральной симметрии, какую эти профили имели бы при теплообмене шара с неподвижным газом. На рис. 4 (по сравнению с рис. 2) очевидно, что при обтекании шара в переходном режиме радиальные профили сильно деформированы в направлении потока, и от упомянутой симметрии уже ничего не осталось. Это обусловлено

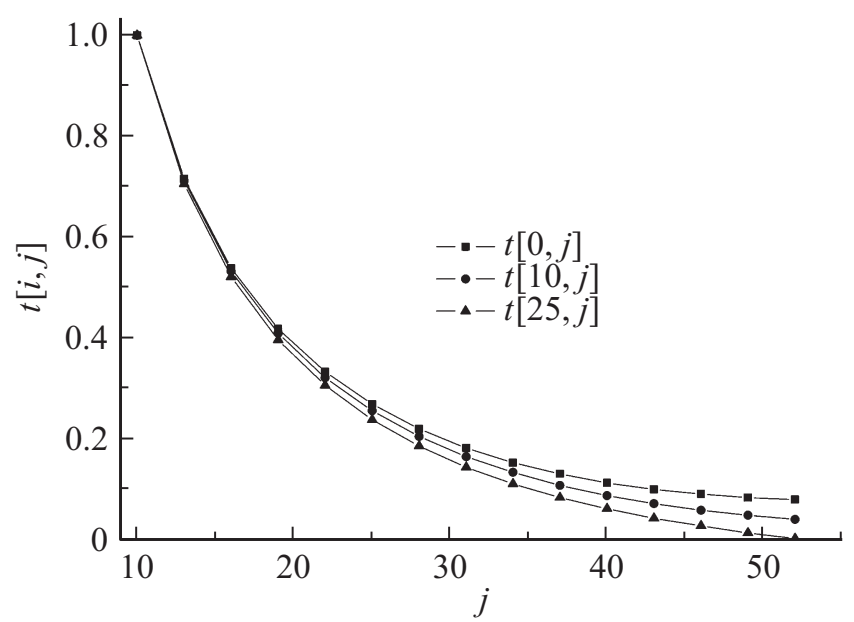

Рис. 2. Радиальные профили температуры газа, полученные для стоксовского режима обтекания шара газом при $\operatorname{Re}=0.125$. 


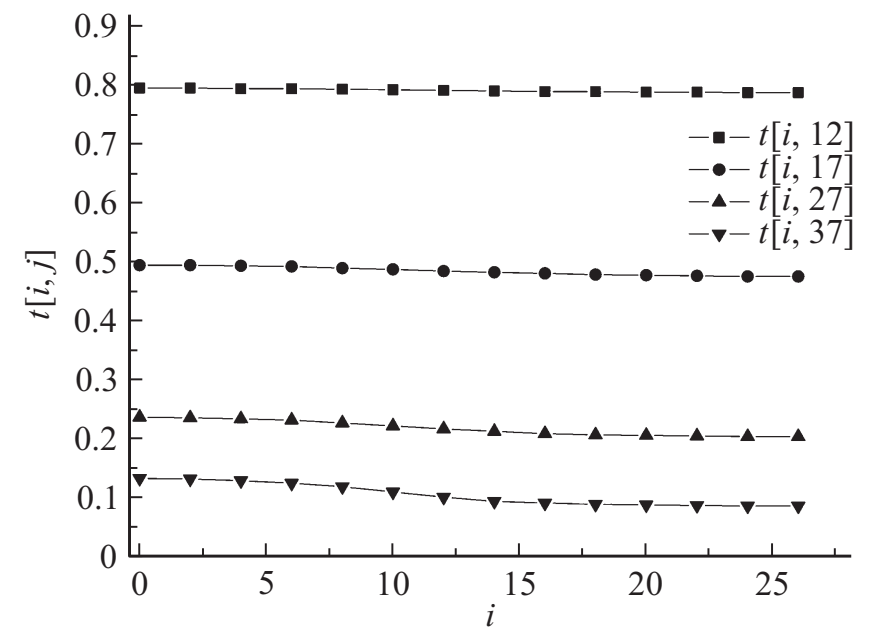

Рис. 3. Профили изменения температуры газа по полярному углу $\theta_{i}$, полученные для стоксовского режима обтекания шара газом при $\mathrm{Re}=0.125$.

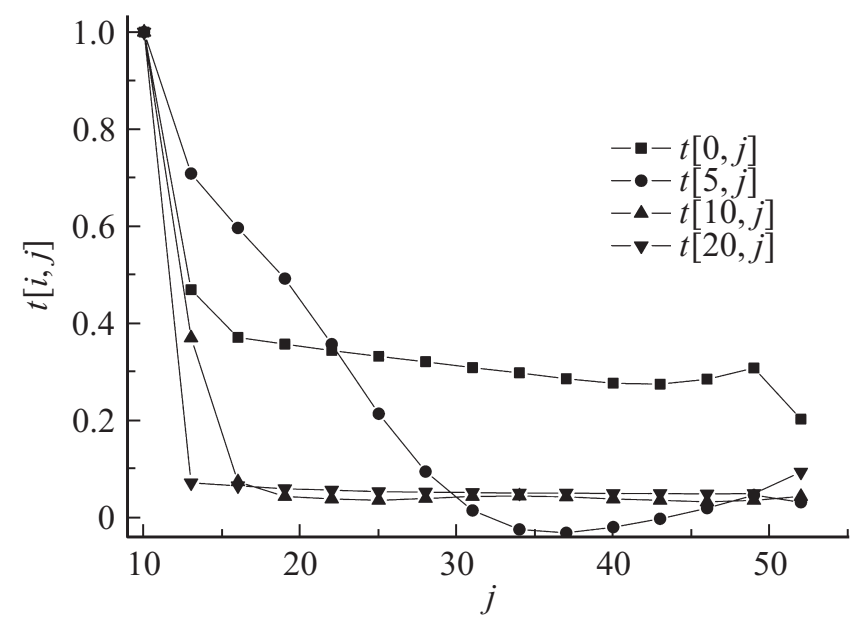

Рис. 4. То же, что на рис. 2 , но для переходного режима обтекания шара газом при $\mathrm{Re}=128$.

изменением характера обтекания шара газом с возрастанием числа Re, в частности, возникновением вблизи „кормовой“ области шара возвратно-вихревого течения, которое надежно и ярко проявилось на профилях скорости газа, приведенных в работе [8].

На рис. 3 очевидно, что в стоксовском режиме обтекания температура $t[i, j]$ газа при $j=$ const мало изменяется по полярному углу $\theta_{i}$. На рис. 5 , напротив, очевидно, что в переходном режиме обтекания изменение температуры $t[i, j]$ газа (при $j=\mathrm{const}$ ) по углу $\theta_{i}$ весьма существенно на разных расстояниях $r_{j}$ от поверхности шара.

Результаты расчетов числа Нуссельта представлены на рис. 6 кружками. Для сравнения в виде кривой приведена известная аппроксимация Ранца-Маршалла [14]

$$
\mathrm{Nu}=2+0.6 \operatorname{Re}^{1 / 2} \operatorname{Pr}^{1 / 3}
$$

Очевидно, рассчитанные по вышеописанной численной модели значения числа $\mathrm{Nu}$ для шара, обтекаемого сво- бодным газовым потоком, во всем исследованном диапазоне значений $0.06<\mathrm{Re}<400$ хорошо согласуются с известными данными. Небольшое различие значений $\mathrm{Nu}$, рассчитанных по модели и формуле (16) при $\mathrm{Re}=1-2$, можно объяснить изменением режима обтекания от стоксовского к переходному, а также ограниченностью числа узлов расчетной сетки.

На рис. 7 и 8 (в разных масштабах) представлены результаты расчетов коэффициента сопротивления $C_{d}$ шара (кружки) и графики известных зависимостей кривыми. Там же ромбами показаны данные эксперимента с каплями распыленной форсункой воды. Очевидно, рассчитанные при $\operatorname{Re}<1$ значения $C_{d}$ для шара, обтекаемого газовым потоком, согласуются с формулой Стокса (2), а рассчитанные при $\operatorname{Re}>2$ - с формулой

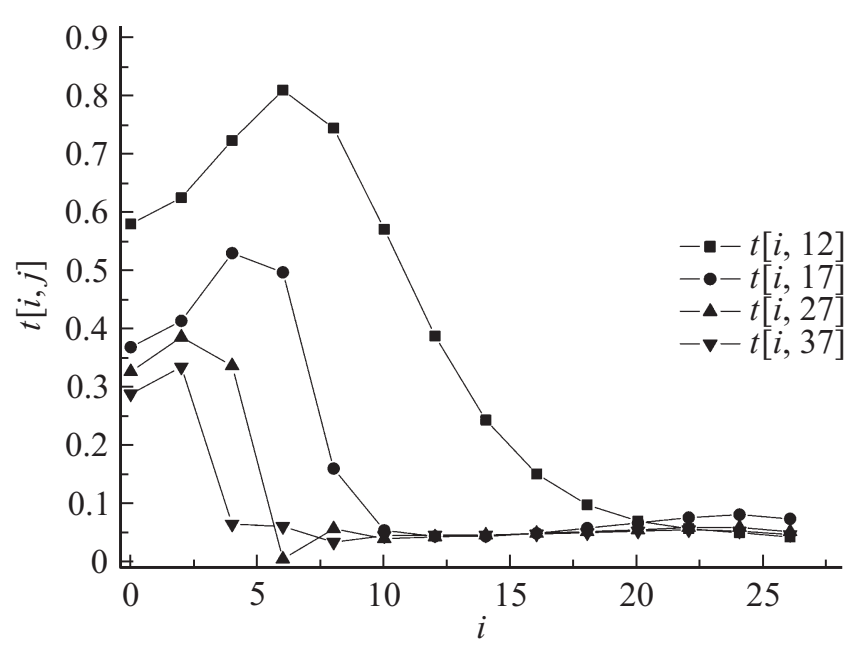

Рис. 5. То же, что на рис. 3, но для переходного режима обтекания шара газом при $\mathrm{Re}=128$.

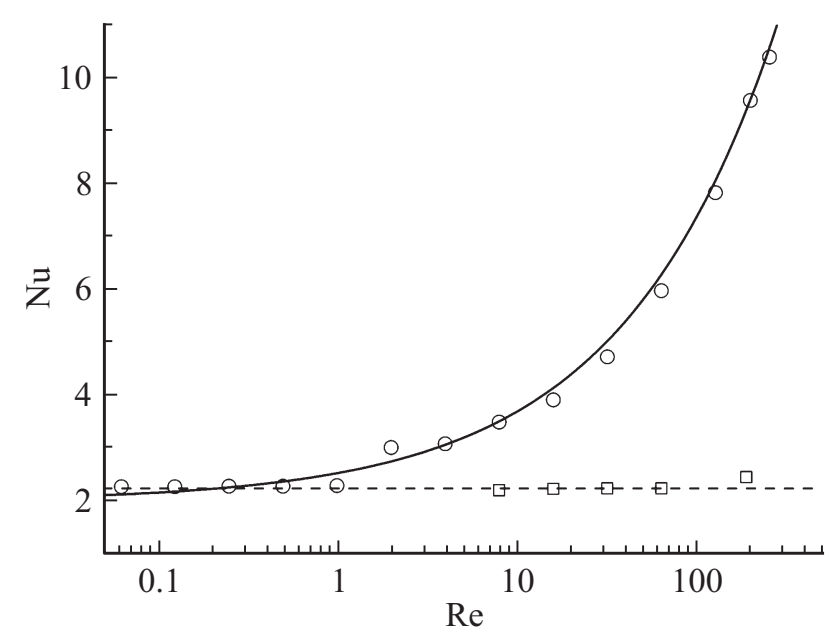

Рис. 6. Зависимости числа Нуссельта $\mathrm{Nu}$ от числа Рейнольдca Re для теплоотдачи шара газу: о - результаты расчета по численной модели обтекания шара ламинарным потоком газа с учетом их теплообмена, $\square-$ то же для обтекания сильнотурбулентным потоком, сплошная кривая - расчет по формуле (16), пунктирная линия - аппроксимация некоторых результатов расчета. 


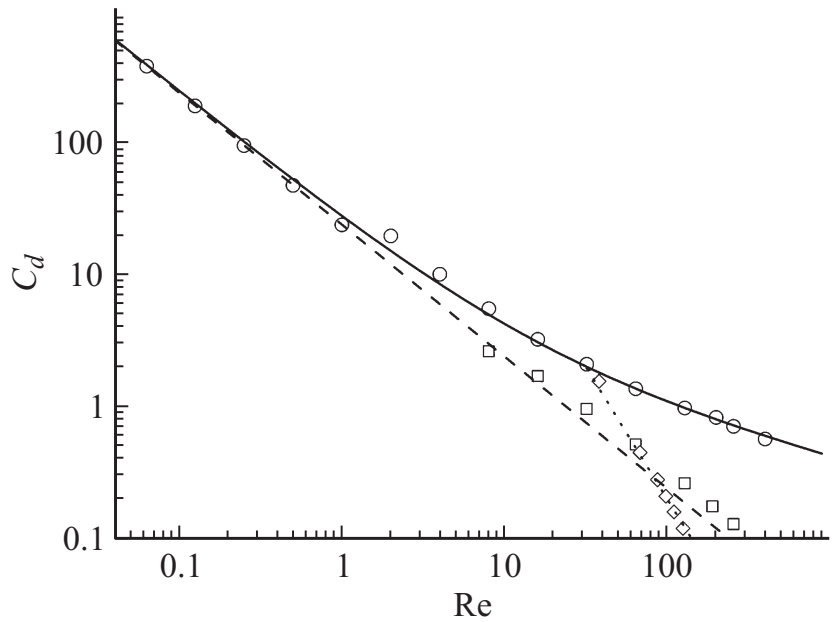

Pис. 7. Зависимости коэффициента сопротивления $C_{d}$ шара от числа Рейнольдса $\operatorname{Re}$ в логарифмическом масштабе: орезультаты расчета по численной модели обтекания шара ламинарным потоком газа с учетом их теплообмена, $\square-$ то же для обтекания сильнотурбулентным потоком, штриховая кривая - расчет по формуле (2), сплошная кривая - расчет по формуле (3), $\diamond$ - эксперимент с каплями воды в факеле форсунки при давлении $P=5 \mathrm{bar}$, пунктирная кривая - аппроксимация этих данных формулой $C_{d}=2000 / \mathrm{Re}^{2}$.

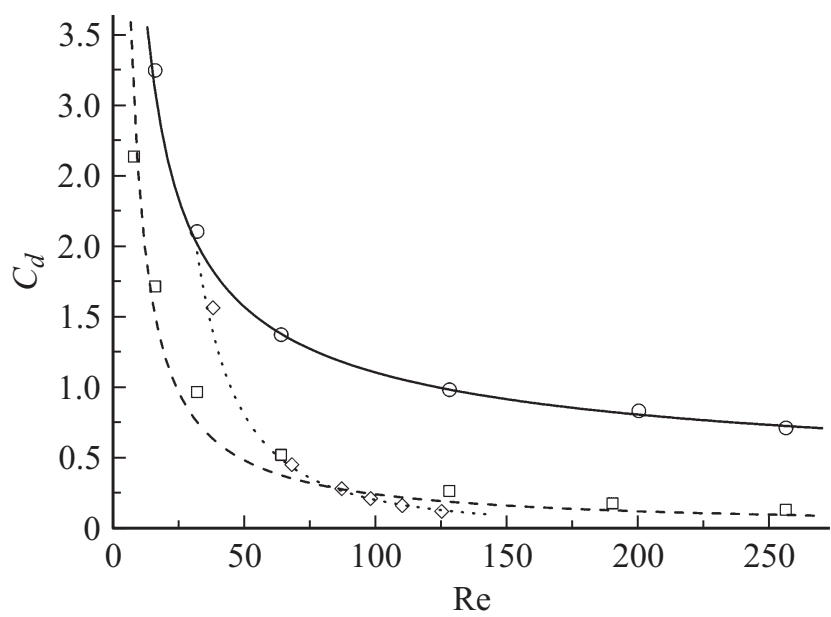

Pис. 8. То же, что на рис. 7, но в другом (линейном) масштабе.

Клячко (3). Небольшое отличие значений $C_{d}$, рассчитанных по предложенной модели и по формулам (2) или (3) при $\operatorname{Re}=1-2$, объясняется теми же причинами, что и для зависимости $\mathrm{Nu}=\mathrm{Nu}(\mathrm{Re})$.

\section{3. Моделирование обтекания шара сильнотурбулентным потоком газа с учетом теплообмена}

При численном моделировании обтекания шара турбулентным потоком использовались следующие соображения и представления.
Если неподвижный шар в переходном диапазоне $\left(\operatorname{Re} \sim 10-10^{2}\right)$ обтекается сильнотурбулентным газовым потоком, например, круглой струей диаметром $D$, то ее можно охарактеризовать числом Рейнольдса $\mathrm{Re}_{1}=$ $=\langle V\rangle D \rho / \mu \sim 10^{5}$ и кинематическим коэффициентом турбулентной вязкости $v_{\tau}$, который можно полагать неизменным в пределах струи и оценить по формулам

$$
\begin{aligned}
v_{\tau} & =\sigma(J / \rho)^{1 / 2}=\sigma(\pi / 4)^{1 / 2} \operatorname{Re}_{1} v=\mathrm{const} \\
& \approx 0.02 \operatorname{Re}_{1} v \sim 2 \cdot 10^{3} v \gg v,
\end{aligned}
$$

где $J=\pi / 4 D^{2} \rho\langle V\rangle^{2}=$ const - поток импульса струи, $\langle V\rangle$ - средняя по сечению струи скорость газа, $\sigma \approx$ $\approx 0.021$ - эмпирическая постоянная [10]. Заметим, что, согласно оценке (17), кинематический коэффициент турбулентной вязкости $v_{\tau}$ в данном случае значительно превосходит аналогичный коэффициент обычной физической вязкости $v$.

Согласно теории „пристенной“ турбулентности, у поверхности обтекаемого тела образуется турбулентный погранслой, в котором перенос импульса определяется суммарным действием $v_{\Sigma}=v_{\tau}(y)+v-$ турбулентной и физической вязкостей [10]. Причем первое слагаемое $v_{\tau}=(0.4 y)^{2}\left|\partial V_{\theta} / \partial y\right|$ изменяется пропорционально квадрату расстояния $y=r-R$ от поверхности шара и на малой толщине $(\delta \ll R)$ погранслоя резко возрастает от нуля до максимального значения, определяемого формулой (17) для удаленной от обтекаемого тела части потока.

Уравнения Рейнольдса для осредненных по времени переменных: скорости, плотности и давления, описывающие квазистационарное течение в турбулентном погранслое, имеют тот же вид, что и уравнения (4), (5), (7), отличаясь заменой $v$ на $v_{\Sigma}$.

В описываемом численном эксперименте использовалась аппроксимация кинематического коэффициента турбулентной вязкости функцией вида

$$
v_{\tau}(y)=v_{\tau}(\infty)(1-R / r)^{2}=2000 v(1-R / r)^{2},
$$

согласно которой $\quad v_{r} \rightarrow 0 \quad$ при $y=(r-R) \rightarrow 0 \quad$ и $v_{\tau} \rightarrow$ const $=2000 v$ при $y \rightarrow \infty$, что соответствует представлениям теории пристенной турбулентности.

Для расчета температуры газа в сильно турбулентном потоке использовалось то же уравнение (10), что и в ламинарном потоке.

В сильнотурбулентном потоке обычно пренебрегают изменением давления в поперечном сечении потока [10]. Если принять, что это верно и в нашем случае, пока нет отрыва погранслоя от поверхности тела, то можно пренебречь первым слагаемым в подынтегральном выражении формулы (15).

На рис. 9 и 10 представлены соответственно радиальные профили температуры газа и профили ее изменения по полярному углу $\theta_{i}$, полученные расчетом при численном моделировании обтекания шара сильнотурбулентным потоком в переходном диапазоне при $\mathrm{Re}=128$. Их 


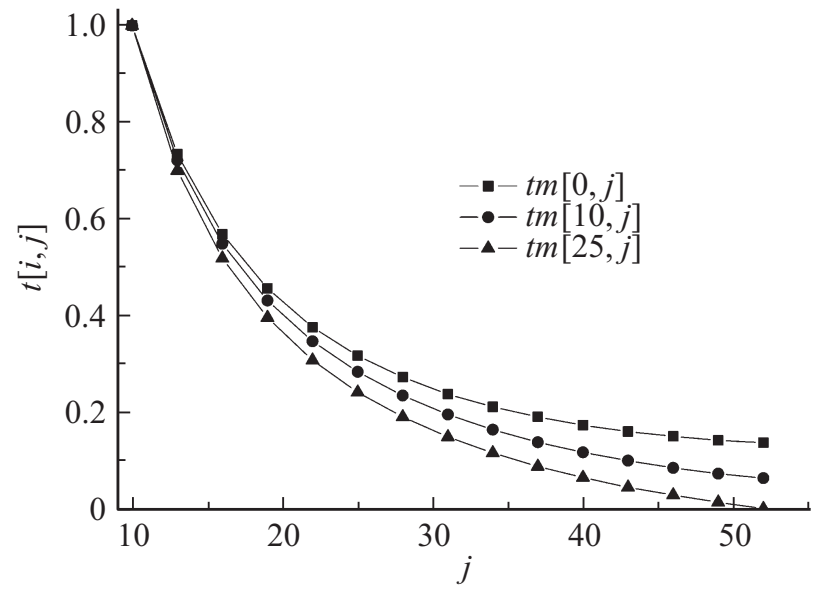

Рис. 9. Радиальные профили изменения температуры газа, полученные расчетом для обтекания шара сильнотурбулентным газовым потоком в переходном режиме при $\mathrm{Re}=128$.

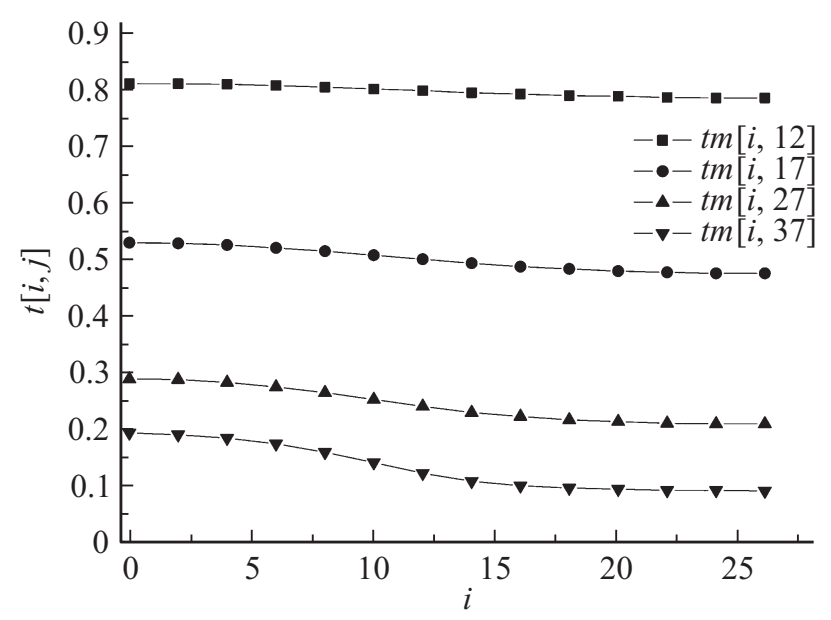

Рис. 10. Профили изменения температуры газа по полярному углу $\theta_{i}$, полученные расчетом для обтекания шара сильнотурбулентным газовым потоком в переходном режиме при $\operatorname{Re}=128$.

сравнение с профилями на рис. 2, 3 свидетельствует об их заметном сходстве (как и для полей скоростей [8]), что обусловлено влиянием сильной турбулентной вязкости газового потока. Напротив, между профилями на рис. 9, 10 и 4, 5 очевидно значительное различие.

Данные расчета коэффициента сопротивления $C_{d}$ шаpa, полученные при численном моделировании его обтекания сильнотурбулентным $\left(\operatorname{Re}_{1} \sim 10^{5}\right)$ потоком, представлены на рис. 7 и 8 квадратиками, расположенными близко к штриховой линии, соответствующей формуле Стокса (2). Там же символами ромбами представлены данные эксперимента [13], полученные для капель воды в факеле распыла форсунки при $P=5$ bar. На рис. 8 , очевидно, при $\operatorname{Re}>50$ результаты эксперимента и расчета для обтекания шара сильнотурбулентным потоком согласуются лучше, чем для обтекания ламинарным потоком. Это подтверждает корректность предложенных модельных представлений о механизме раннего кризиса сопротивления тела в сильнотурбулентном потоке.

Большее различие при $\operatorname{Re}<50$ между данными эксперимента и расчета можно объяснить тем, что в условиях эксперимента в этой области значений числа $\mathrm{Re}$, соответствующей меньшим расстояниям от форсунки [3], степень турбулентности газового потока была еще недостаточной для возникновения раннего кризиса сопротивления капель.

\section{4. Ранний кризис теплоотдачи}

В работах $[7,8]$ приведено объяснение раннего кризиса сопротивления шара влиянием изначально сильной турбулентности набегающего на него потока. Большая турбулентная вязкость $v_{\tau}$ потока создает условия обтекания и профили осредненных по времени скоростей газа сходные со стоксовским обтеканием при $\operatorname{Re}<1$. А малая (по сравнению с $v_{\tau}$ ) величина физической вязкости $v$, которая играет основную роль вблизи поверхности шара, уменьшает его коэффициент сопротивления $C_{d}$ в несколько раз.

Как отмечалось во Введении, с учетом аналогии между явлениями переноса импульса, тепла и массы примеси кризис сопротивления шара в сильнотурбулентном потоке с неизбежностью должен влиять на тепло- и массообмен шара с газом. В работе [11] сделана попытка оценить это влияние следующим образом.

Для связи параметров $\alpha d / \lambda=\mathrm{Nu}(\mathrm{Re}, \mathrm{Pr})$ конвективного теплообмена сферической капли с газовым потоком известно из [14] уравнение Ранца-Маршалла (16), которое упрощается с учетом того, что для газов $\operatorname{Pr}^{1 / 3} \approx 1$.

При кризисе сопротивления улучшается обтекание шарообразной капли, приближаясь к стоксовскому, при этом число $\mathrm{Nu}(\mathrm{Re}) \rightarrow \mathrm{Nu}(0)=2$. В переходном диапазоне чисел $\mathrm{Re}$, где возникает ранний кризис сопротивления капель, согласно (16): $\mathrm{Nu}_{1}=7$ при $\operatorname{Re}_{1}=$ $=70$ и $\mathrm{Nu}_{2}=11.5$ при $\operatorname{Re}_{2}=250$. Тогда отношения $\mathrm{Nu}_{1} / \mathrm{Nu}(0) \approx 3.5, \mathrm{a} \mathrm{Nu}_{2} / \mathrm{Nu}(0) \approx 5.8$.

Таким образом, интенсивность теплообмена капли с газом при раннем кризисе сопротивления должна уменьшаться в 3.5-5.8 раза, т.е. примерно так же, как коэффициент $C_{d}$ гидродинамического сопротивления капли.

Оказалось возможным проверить эту оценку аналогично работе [8] - путем численного моделирования обтекания шара сильнотурбулентным газовым потоком с учетом теплообмена шара с газом. Для этого описанная в [8] гидродинамическая модель была дополнена уравнением (10), учитывающим теплообмен шара с газом.

На рис. 6 квадратиками показаны результаты расчета числа Нуссельта $\mathrm{Nu}$ в зависимости от числа Рейнольдca $\operatorname{Re}$ по представленной выше модели обтекания шара сильнотурбулентным потоком газа с учетом их теплообмена. Очевидно, в переходном диапазоне чисел $\mathrm{Re}=$ $=8-200$ значения числа $\mathrm{Nu}$ мало отличаются от тех, что свойственны стоксовскому обтеканию шара ламинарным потоком и примерно равны 2.2. Это обстоятельство отмечено на рис. 6 штриховой линией. 


\section{Заключение}

Таким образом, описанный в разд. 1 и 2 настоящей работы численный эксперимент показал, что, вопервых, предложенный алгоритм расчета обтекания шара ламинарным газовым потоком с учетом теплообмена позволяет вполне удовлетворительно рассчитывать поля скоростей, давлений и температуры газа, силу и коэффициент сопротивления шара, поток тепла и коэффициент теплоотдачи шара обтекающему газовому потоку. Результаты расчета хорошо согласуются с известными данными.

Во-вторых, сочетание предложенного алгоритма с элементами теории пристенной турбулентности позволило смоделировать обтекание шара сильнотурбулентным потоком, рассчитать и в этом случае поля скоростей и температур газа. А также позволило рассчитать коэффициент сопротивления $C_{d}$ шара (в согласии с экспериментальными данными для раннего кризиса сопротивления) и характеризующее теплоотдачу шара число Нуссельта $\mathrm{Nu}$, значения которого оказались заниженными в несколько раз по сравнению с $\mathrm{Nu}$ для ламинарного обтекании шара в переходном режиме.

Это подтвердило правильность сделанного в работе [11] предположения о возможности возникновения раннего кризиса теплоотдачи, которым, как оказалось, сопровождается кризис сопротивления.

\section{Список литературы}

[1] Броунштейн Б.И., Фишбейн Г.А. Гидродинамика, массои теплообмен в дисперсных системах. Л.: Химия, 1977. $280 \mathrm{c}$.

[2] Torobin L.B., Gauvin W.H. // Can. J. Chem. Eng. 1959. Vol. 37. N 4. P. 129-141.

[3] Шлихтинг Г. Теория пограничного слоя / Пер. с нем. М.: Наука, 1974. 712 с.

[4] Симаков Н.Н. // ЖТФ. 2004. Т. 74. Вып. 2. С. 46-51.

[5] Simakov N.N., Simakov A.N. // J. Appl. Phys. 2005. Vol. 97. P. 114901.

[6] Симаков Н.Н. // ЖТФ. 2010. Т. 80. Вып. 7. С. 1-7.

[7] Симаков Н.Н. // ЖТФ. 2011. Т. 81. Вып. 11. С. 23-30.

[8] Симаков Н.Н. // ЖТФ. 2013. Т. 83. Вып. 4. С. 16-20.

[9] Ландау Л.Д., Лифиии, Е.М. Теоретическая физика, T. IV. Гидродинамика. М.: Наука, 1988. 736 с.

[10] Лойиянский Л.Г. Механика жидкости и газа. М.: Наука, 1978. $736 \mathrm{c.}$

[11] Симаков Н.Н. // Тр. Междунар. научно-технической конф. Проблемы ресурсо- и энергосберегающих технологий в промышленности и АПК. / Под ред. проф. В.Н. Блиничева. Иваново: ИГХТУ, 2014. Т. 2. С. 389-395.

[12] Федоренко Р.П. Введение в вычислительную физику. М.: Изд-во МФТИ, 1994. 528 с.

[13] Поттер Д. Вычислительные методы в физике / Пер. с англ. М.: Мир, 1975. 392 с.

[14] Ranz W.E., Marshall W.R. // Chem. Eng. Progress, 1952. Vol. 48. N 5. P. 173. 